\title{
BMJ Open Metabolically healthy obesity and risk of cardiovascular disease, cancer, and all-cause and cause-specific mortality: a protocol for a systematic review and meta-analysis of prospective studies
}

\author{
Simiao Tian, ${ }^{1}$ Yazhuo Liu, ${ }^{2}$ Ao Feng, ${ }^{2}$ Keli Lou, ${ }^{2}$ Huimin Dong ${ }^{2}$
}

To cite: Tian S, Liu Y, Feng A, et al. Metabolically healthy obesity and risk of cardiovascular disease, cancer, and all-cause and cause-specific mortality: a protocol for a systematic review and meta-analysis of prospective studies. BMJ Open 2019;9:e032742. doi:10.1136/ bmjopen-2019-032742

- Prepublication history for this paper is available online. To view these files, please visit the journal online (http://dx.doi. org/10.1136/bmjopen-2019032742).

Received 03 July 2019 Revised 26 September 2019 Accepted 11 October 2019

A) Check for updates

(c) Author(s) (or their employer(s)) 2019. Re-use permitted under CC BY-NC. No commercial re-use. See rights and permissions. Published by BMJ.

${ }^{1}$ Department of Scientific Research Project, Affiliated Zhongshan Hospital of Dalian University, Dalian, China ${ }^{2}$ Department of Clinical Nutrition and Metabolism, Affiliated Zhongshan Hospital of Dalian University, Dalian, China

Correspondence to

Dr Simiao Tian;

simiao_tian@sina.com

\section{ABSTRACT}

Introduction Metabolically healthy obese phenotype (MHO) refers to obese individuals with an adequate metabolic profile and absence of metabolic syndrome. Many prospective studies have reported the benign condition relating the $\mathrm{MHO}$ phenotype and its potential role in reducing risk of cardiovascular disease, total cancer, and all-cause and cause-specific mortality. However, inconsistent results were found and the question remains controversial. We aim to conduct a systematic review and meta-analysis to clarify the associations these associations from relevant prospective studies. Methods and analysis The Preferred Reporting Items for Systematic Reviews and Meta-Analyses for Protocols 2015 statement was used to prepare this protocol. MEDLINE, Web of Science databases, EMBASE and Cochrane Database will be used for literature search from their inception up to December 2019 with restriction of published studies in English. Published prospective studies reporting adjusted relative risk (RR) estimates for the association between MHO phenotype and cardiovascular disease, total cancer, all-cause or cause-specific mortality will be included. The process of study screening, selection and data extraction will be performed independently by two reviewers, and the risk of bias for the studies included will be assessed using the Newcastle-Ottawa Quality Assessment Scale. HRs or RRs for disease events and mortality with $95 \%$ Cls will be considered as primary outcomes, and summary HRs/ RRs will be pooled using random-effects models. The Cochrane's $Q$ and the $\left.\right|^{2}$ statistics will be used to assess and quantify heterogeneity, respectively. Subgroup analysis will also be carried out according to study characteristics to investigate potential sources of heterogeneity.

Ethics and dissemination As this meta-analysis is performed based on the published studies, no ethical approval and patient safety considerations are required. The findings of the study will be reported and submitted to a peer-reviewed journals for publication. PROSPERO registration number CRD42019121766.

\section{INTRODUCTION}

Obesity is now one of the major public health problems and becomes a worldwide epidemic
Strengths and limitations of this study

- This review is anticipated to be the first comprehensive meta-analysis of prospective studies to address the metabolically healthy obesity (MHO) to the risk of coronary heart disease, stroke, cardiovascular disease, total cancer and all-cause mortality as well as less common causes of death.

- This systematic review and meta-analysis will provide a more up-to-date and comprehensive assessment of the MHO and several health outcomes.

- This meta-analysis has a comprehensive literature search strategy involving restriction of studies to prospective studies, and will ensure that both the risk of bias and the quality of evidence of the included studies is properly assessed by Cochrane risk of bias assessment tool and Newcastle-Ottawa Quality Scale, respectively.

- Only included studies written in English may lead to publication bias.

in the past four decades. Its prevalence has risen globally from $3.2 \%$ to $10.8 \%$ in adult men and from $6.4 \%$ to $14.9 \%$ in adult women in the same period. ${ }^{1}$ The excess body weight was estimated to affect nearly 2 billion people, and accounted for approximately 4 million deaths and 120 million disability-adjusted life years. ${ }^{23}$ Obesity is a well-established risk factor for a great number of cardiovascular diseases (CVDs) and metabolic disorders, ${ }^{4-6}$ and also has been shown as the main cause of CVD, cancer mortality and all-cause mortality. ${ }^{7-10}$ However, obese people may vary in their body fat distribution and cardiometabolic profiles, thereby their association with morbidity and mortality could be heterogeneous in the obese people. ${ }^{11} 12$ In this context, recent search focused on a novel subgroup of obese individuals who seem to have an adequate metabolic profile and do not have metabolic syndrome (MetS)while being categorised as 
obese, referred as metabolically healthy obese $(\mathrm{MHO}) .^{1314}$ Multiple studies showed that MHO phenotype accounted for as much as $10 \%-50 \%$ of the obese adults, depending on the population and the criteria used to ascertain metabolic health. ${ }^{12}{ }^{15} \mathrm{~A}$ very recent meta-analysis of 40 population-based studies reported an overall prevalence of $35 \%$ among obese adults. ${ }^{16}$

The extent to which the MHO phenotype is the benign condition and is associated with a lower risk of adverse health outcomes and all-cause mortality remains controversial. Some studies have confirmed a protective effect and no increased risk of CVD and mortality among MHO individuals, particularly compared with at-risk obese $^{17-19}$; whereas several other studies have shown a higher risk of CVD, cancer incidence and mortality in this group compared with metabolically healthy normal weight (MHNW) individuals. ${ }^{20-22}$ For instance, a 10-year follow-up study of 25626 women aged 45 years and more found no increased CVD risk for MHO individuals, ${ }^{23}$ a finding replicated in a 15-year follow-up Italian study of obesity and insulin sensitivity. ${ }^{24}$ In contrast, another study showed that overweight and obese individuals without the MetS had an increased risk of CVD compared with MHNW individuals after a 17-year follow-up, a finding justified by using five different metabolic health definitions. ${ }^{25}$ It is important to note that inconsistent results depend on study design, population, follow-up time and MHO definition used. Several meta-analysis studies have investigated this ongoing controversy ${ }^{26-28}$; however, the reliability of summarised evidences was questionable due to methodological constraints. Some roughly merged the incidents of CV events and all-cause mortality together instead of differentiating these two outcomes, some calculated the pooled risk estimate based on unadjusted risk estimates, and some only considered MetS as MHO definitions, resulting in a limited number of analysed studies. ${ }^{2627}$

Another two recent meta-analyses reported that, compared with participants with MHNW, those with MHO were at higher risk of cardiovascular events but not all-cause mortality. ${ }^{29}$ The systematic review and meta-analysis by Eckel et al is particularly important because it was the first to carefully consider the full range of possible definitions of metabolic health, ${ }^{28}$ and this aspect is crucial when addressing the role of this complex condition for the prediction and prevention of cardiometabolic diseases and possibly of certain types of cancer. ${ }^{30}$ Besides, the meta-analysis conducted by Eckel et al extended literature search to include only prospective studies with strict standard of reference groups considered, and perform a comprehensive subgroup analyses. ${ }^{28}$ The meta-analysis conducted by Zheng $e t ~ a l,{ }^{29}$ including a large sample size, enabled the determination of a robust and reliable risk estimates of $\mathrm{CV}$ events and mortality for MHO individuals by using both raw data and fully adjusted effect sizes from original studies, but these two aforementioned meta-analysis study were not up to date, with their literature search until April 2014 and
September 2015, respectively, and since then, according to our general search, there are more than 17 new publications investigating $\mathrm{MHO}$ and health outcomes between 2016 and 2019. More importantly, the association with cancer events and various cause-specific mortality is still scarce.

\section{OBJECTIVES}

The protocol study is designed to establish an explicit methodology for systematically and comprehensively conducting a review evidence and meta-analysis, and the aim is to (1) clarify whether is there an increase in risk of developing CVD, total cancer, and all-cause and cause-specific mortality in adults with MHO, compared with their MHNW peers and (2) to define more accurate estimates of risk.

\section{METHODS AND ANALYSIS \\ Registration and review design}

The procedure for this study will be conducted in accordance with the guidelines provided by the Preferred Reporting Item for Systematic Reviews and Meta-analyses Protocols (PRISMA). ${ }^{31}$

\section{Search strategy}

A literature search will be undertaken using the following electronic databases: MEDLINE (via PubMed), ISI Web of Knowledge databases, EMBASE and the Cochrane Database to identify published studies. The databases will be searched from their inception to December 2019. In addition, the literature search will be later updated and supplemented through the manual review of reference list of obtained articles. The following search terms will be used as keywords or (and) medical subject headings $(\mathrm{MeSH})$ terms in the electronic search: body mass index (BMI), obesity, metabolic, metabolically, healthy, MetS, CVD, risk, mortality, cause of death. Details of search terms and strategy for MEDILINE are provided in table 1, and this strategy will be adapted to suit other databases.

\section{Inclusion criteria}

All prospective studies of $\mathrm{MHO}$ and incidence or mortality from coronary heart disease (CHD), stroke, CVD, total cancer, and all-cause and cause-specific mortality will be considered eligible and included if they meet the following criteria:

1. The study design is a prospective cohort study;

2. MHO and other obesity phenotypes (eg, MHNW and metabolically unhealthy obese (MUO)) are defined according to the cross-classification of obesity criteria and metabolic health status. Obesity is defined using BMI, waist circumference (WC) or body fat per cent; metabolic health status is defined using any of the following published MetS criteria: the Adult Treatment Panel-III (ATP-III)-based criterion (including any extended or modified ATP-III criteria), the Internation- 
Table 1 Proposed search terms

\section{Search items}

1. "Body mass index" OR "Obesity.mp. or OBESITY/" OR "Adiposity.mp. or ADIPOSITY/" OR "Waist Circumference.mp. or Waist Circumference/" OR "body fat or adipose tissue"

2. "Metabolic Syndrome.mp. or Metabolic Syndrome/" OR "Insulin Resistance.mp. or Insulin Resistance/" OR "Insulin sensitive.mp." OR "Metabolic Health.mp." OR "Metabolically Healthy.mp." OR "Obesity/ or Metabolically Benign.mp. or Overweight/" OR "Metabolically Healthy Obesity.mp. or Obesity, Metabolically Benign/" OR "Metabolically Benign Obesity.mp. or Obesity, Metabolically Benign/"

3. \#1 AND \#2

4. "coronary heart disease" or "heart disease" or "ischemic heart disease" or "ischaemic heart disease" or "CHD" or "coronary artery disease" or "myocardial infarction" or "stroke" or "ischemic stroke" or "haemorrhagic stroke" or "cardiovascular disease" or CVD or cancer or "total cancer" or mortality or "all-cause mortality" or "total mortality" or "survival"

5. "case-control" or "cohort" or "cohorts" or "prospective" or "longitudinal" or "retrospective" or "follow-up" or "crosssectional" or "population-based" or "relative risk" or "relative risk" or "odds ratio" or "hazard ratio" or "incidence rate ratio"

\section{6. \#3 AND \#4 AND \#5}

al Diabetes Federation (IDF) criteria, Joint Interim Statement (JIS) criteria, Harmonised MetS criteria, the Wildman criteria, the Karelis criteria, insulin resistance (IR) —or risk score-based criteria (eg, the Homoeostasis model assessment of IR (HOMA-IR) index of having HOMA-IR in the upper quartile of the HOMA index and the triglyceride-glucose (TyG) index of having TyG >8.82/8.73 for men/women) or other cardiometabolic clusterings.

3. The main outcomes of interest are CHD (total CHD or major coronary event, non-fatal myocardial infarction (MI), any MI, fatal MI, incident ischaemic heart disease, fatal ischaemic heart disease, acute coronary syn-

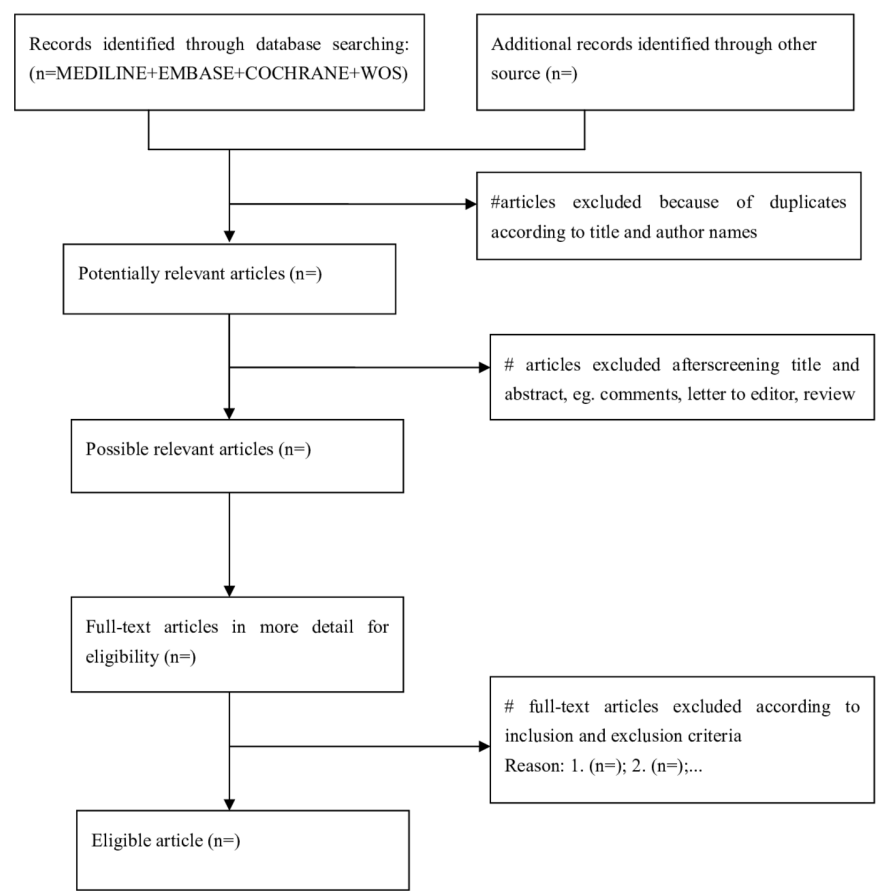

Figure 1 The Preferred Reporting Items for Systematic Reviews and Meta-Analyses flow chart of study selection. drome), stroke (total stroke, ischaemic, haemorrhagic, intracerebral and subarachnoidal haemorrhage), total CVD (CHD and stroke combined), and total cancer and all-cause mortality; the secondary outcomes will be cause-specific mortality from any cause of death.

4. Outcomes are measured by multivariate Cox proportional hazards models, and the relative ratio (RR) or HR and the corresponding $95 \%$ CI are reported.

5. Population of adults or participants are aged 18 years and older.

6. Studies are published in English.

\section{Study selection}

All investigators will be properly trained prior to data screening task. Two review author (KL and HD) will first screen the title and abstract of the searched studies independently and in duplicate to assess the eligibility of the searched studies. Then, all potentially eligible studies will be retrieved and the same review authors will review full-text articles for inclusion, according to prespecified inclusion criteria. When disagreements occur, it will be resolved by group discussion or, if required, a third author (AF) will be consulted to evaluate the full text and the discrepancy. In addition, excluded studies and the rationale for exclusion will be documented. Figure 1 depicts the study selection processes in a PRISMA flow diagram.

\section{Data extraction}

We will extract results and study characteristics into tables using a standardised data collection form from eligible studies. Information that needed to be extracted will be as follow: first author's name, year of publication, country or region, duration of follow-up, study location, sample size and number of events or deaths, gender proportion and age at baseline year, baseline MHO sample size, MHO definition, adjustments or covariates in the models, outcomes, the size of the association (HRs, RRs or ORs with $95 \% \mathrm{CI})$. We compared the risk of having various 
health outcomes, such as mortality and CVD events, and calculate the pooled risk estimates for the MHO, metabolically unhealthy normal weight (MUNW) and MUO phenotypes using MHNW participants as the reference. If one article contained several obesity and metabolic health definitions, we will treat each definition as an independent one. It is noteworthy mentioning that several studies revealed that MUNW individuals, even though with a normal BMI range, was unexpected associated with higher risk of all-cause mortality and/or cardiovascular events. ${ }^{26}$ In this regard, Stefan et al provided a comprehensive review and data addressing to what extent major risk phenotypes determine metabolic health in lean compared with overweight and obese people and provide support for the existence of a lipodystrophy-like phenotype in the general population. ${ }^{32}$ Therefore, for the sake of integrity of the study, the risk of MUNW and other obesity phenotypes with health outcomes will also be summarised in the present study.

The data extraction will be independently conducted by $\mathrm{KL}$ and $\mathrm{HD}$, and be checked for accuracy by AF. All disagreements will be settled by discussion until a consensus is reached. In case of lacking key information, authors of primary studies will be contacted and consulted for obtaining missing data.

\section{Study quality assessment}

Study quality of included studies will be assessed by the Newcastle-Ottawa Quality Scale (NOS) adopted for cohort studies, ${ }^{33}$ and this scale awards $0-9$ score points based on the selection, comparability and outcome assessment. Specifically, the NOS includes the following criteria with associated points: (1) representativeness $(*)$; (2) selection of non-exposed cohort (*); (3) exposure-ascertainment $(*) ;(4)$ demonstration of outcome not present at start $(*) ;(5)$ adjustment for age/adjustment for any other factor $(* *) ;(6)$ assessment of outcome $(*) ;(7)$ long enough follow-up $(*)$ and (8) adequacy of follow-up (*). We will consider studies with 0-3, 4-6 and 7-9 points to represent low, medium and high-quality studies, respectively. The study quality will be independently assessed by two reviewers (KL and HD), and if any discrepancies, we will resolved by group discussion or consultation from with a third reviewer.

\section{Data synthesis and statistical analysis}

Once the data extraction has been completed, we will conduct the statistical analysis. All statistical analyses will be done with R V.3.2 software (R Foundation for Statistical Computing, Vienna, Austria) ${ }^{34}$ and 'metafor' package of $\mathrm{R} .{ }^{35}$ In the present meta-analysis, the HR with its $95 \%$ CI will be as a common measure of incidence or mortality from CHD, stroke, total CVD and total cancer, and of all-cause mortality for the MHO group compared with the MHNW group (the reference group). For studies that reported several multivariable-adjusted HR, we will use the most fully adjusted for potential confounders in the meta-analysis. Heterogeneity between studies will be evaluated using the Cochrane's $\mathrm{Q}$ and Higgins $\mathrm{I}^{2}$ statistics, respectively. ${ }^{36}$ For the $Q$ statistic, a $\mathrm{p}<0.1$ is considered to be significant, and $\mathrm{I}^{2}$ values of $0,25,50 \%$ and $75 \%$ represent no, low, moderate and high heterogeneity, respectively. Either fixed-effects or random-effects models, depending on heterogeneity magnitude, will be applied to calculate the summary risk estimates and 95\% CI for outcomes in the MHO group. In the fixed-effect model, the pooled HR is obtained by averaging the $\operatorname{lnHR}$ (HR value in $\log$ scale) weighted by the inverses of their variances. ${ }^{37}$ In the random-effect model, the DerSimonian-Laird method is used to further incorporate between-study heterogeneity. ${ }^{38}$

The sensitivity analyses will also performed when MetS was used for metabolic health criteria. In literature, several studies defined metabolic health by the absence of all metabolic factors, and this stricter definition may lead a different conclusion. ${ }^{28}$ This findings were consistent with a very recent evidence based on the large European Prospective Investigation into Cancer and Nutrition study (CVD) ${ }^{39}$ In this case-cohort analysis including 520000 Europeans after a median follow-up of 12.2 years, Lassale et al found that the MHO phenotype, defined as none of MetS component, was not associated with increased risk of CHD (HR, 1.21, 95\% CI 0.76 to 1.92 ) whereas $\mathrm{MHO}$ individuals were at higher risk of CHD with loose definition of MetS (HR 1.28, 95\% CI 1.03 to 1.58$).{ }^{39}$ For the sake of the integrity of the study and comparability with other meta-analysis, we will also perform additional sensitivity analyses with different definitions of metabolic health when MetS criteria were used: excluding the WC criterion from the definition of MetS, modifying the definition of $\mathrm{MH}$ to be <2 abnormalities; and defining $\mathrm{MH}$ participants as having none of four possible abnormalities (elevated blood pressure, triglyceridaemia, hyperglycaemia, low HDL cholesterol).

\section{Subgroup analyses}

In case of substantial heterogeneity, subgroup analyses will be further performed to investigate the potential source of between-study heterogeneity using following variables: gender (men and women), model adjusted for physical activity (PA) (no vs yes), follow-up duration $(<5$, $5-10$ and $>10$ years $)$, participant's age at baseline $(<50$ and $\geq 50$ years), criterion used to define metabolic health (ATP-III, JIS or IDF, HOMA vs others), geographical location (Asia, Europe, North America, others), sample size $(<5000,5000-10000,>10000)$ and study quality $(0-3$ stars, $4-6$ stars, $7-9$ stars).

It is noteworthy mentioning that among various factors, PA and/or cardiorespiratory fitness (CRF) has been recognised as a novel characteristic of the MHO, as well as play an important role in MHO prognosis. ${ }^{40}$ Specially, based on Aerobics Center Longitudinal Study, ${ }^{40}$ MHO individuals have a significantly higher CRF level than the individuals with MUO, and these findings have been confirmed by recent meta-analysis of Ortega et al that MHO, compared with MUO, have higher levels of 
PA, lower levels of sedentary behaviour and higher levels of $\mathrm{CRF}^{41}{ }^{41}$ Recently, Lavie et al provided a state-of-the-art review on the causes of obesity and effective modalities for this prevention, and the importance of fitness and lifestyle consideration to protect MHO from CVDs. ${ }^{40}$ Therefore, the impact of PA/CRF will be taken into account in the subgroup analysis for the prognosis of future all-cause mortality and other health outcomes in MHO individuals compared with MHNW.

It is also important to recognise that follow-up duration is a critical element in evaluating low-risk populations for the future events. Several studies observed that an increased risk of adverse clinical outcomes occurred only after 8-10 years of follow-up, ${ }^{42}{ }^{43}$ which suggests a transient nature of the MHO phenotype. Indeed, based on a large-scale Nurses' Health Study including 90257 women, Eckel et al found that after 30-year follow-up, the majority of MHO converted to unhealthy phenotypes, and among those who maintained MHO status during follow-up were still at a higher CVD compared with their MHNW peers (HR $1.57,95 \%$ CI 1.03 to 2.38$).{ }^{44}$ In this regard, whether or not MHO is a benign obesity phenotype may be impacted on the length of follow-up; thus, the duration of follow-up is another important factor to take into account in the subgroup analysis.

Furthermore, a sensitivity analysis will be conducted by removing one study in each turn, the rest of the studies are analysed to investigate the robustness of the findings. ${ }^{45}$ Potential publication bias will be assessed with the aid of the Egger's rank and regression test, ${ }^{4647}$ and the visual assessment of funnel plots will also be used if there are sufficient studies (10 or more) in the meta-analysis. ${ }^{48}$

\section{Patient and public involvement}

Patients and/or the public were not directly involved in the design or planning of the study.

\section{Potential protocol amendments}

The current protocol as written will not be modified in the course of the study. However, any modification will be concisely described in the final review.

\section{DISSEMINATION}

The findings of the study will be reported according to the PRISMA-compliant guidelines and submitted to a peer-reviewed journals for publication and also presented at conferences.

Acknowledgements The authors would like to thank the experts from Native EE for invaluable assistance in English language editing.

Contributors ST designed the study protocol and registered the protocol on the PROSPERO database. YL and AF drafted the manuscript. $K L$ and $H D$ tested the feasibility of the study. AF will perform the data collection and analyses. ST revised and finalised the study protocol. All authors reviewed and approved the final manuscript for submission.

Funding This research was funded by National Natural Science Foundation of China (81803329) and China Postdoctoral Science Foundation (2018M631780).

Competing interests None declared.
Patient consent for publication Not required.

Ethics approval This study will not conduct a primary data collection, but will only include previous published studies. Therefore, no ethical approval will be required.

Provenance and peer review Not commissioned; externally peer reviewed.

Open access This is an open access article distributed in accordance with the Creative Commons Attribution Non Commercial (CC BY-NC 4.0) license, which permits others to distribute, remix, adapt, build upon this work non-commercially, and license their derivative works on different terms, provided the original work is properly cited, appropriate credit is given, any changes made indicated, and the use is non-commercial. See: http://creativecommons.org/licenses/by-nc/4.0/.

\section{REFERENCES}

1 NCD Risk Factor Collaboration (NCD-RisC). Trends in adult bodymass index in 200 countries from 1975 to 2014: a pooled analysis of 1698 population-based measurement studies with $19 \cdot 2$ million participants. Lancet 2016;387:1377-96.

2 Afshin A, Forouzanfar MH, Reitsma MB, et al. Health effects of overweight and obesity in 195 countries over 25 years. N Engl J Med 2017:377:13-27.

3 Swinburn BA, Kraak VI, Allender S, et al. The global Syndemic of obesity, undernutrition, and climate change: the Lancet Commission report. Lancet 2019;393:791-846.

4 Janssen I, Katzmarzyk PT, Ross R. Waist circumference and not body mass index explains obesity-related health risk. Am J Clin Nutr 2004;79:379-84.

5 Lu Y, Hajifathalian K, Ezzati M, et al. Metabolic mediators of the effects of body-mass index, overweight, and obesity on coronary heart disease and stroke: a pooled analysis of 97 prospective cohorts with 1.8 million participants. Lancet 2014;383:970-83.

6 Song Z-Z, Wang J, Zhang J. Body mass index, central obesity, and mortality among coronary disease subjects. J Am Coll Cardiol 2013;62:85

7 Meigs JB, Wilson PWF, Fox CS, et al. Body mass index, metabolic syndrome, and risk of type 2 diabetes or cardiovascular disease. $J$ Clin Endocrinol Metab 2006;91:2906-12.

8 Murphy N, Cross AJ, Abubakar M, et al. A nested Case-Control study of metabolically defined body size phenotypes and risk of colorectal cancer in the European prospective investigation into cancer and nutrition (EPIC). PLoS Med 2016;13:e1001988.

9 Spahillari A, Mukamal KJ, DeFilippi C, et al. The association of lean and fat mass with all-cause mortality in older adults: the cardiovascular health study. Nutr Metab Cardiovasc Dis 2016;26:1039-47.

10 Park Y-MM, White AJ, Nichols HB, et al. The association between metabolic health, obesity phenotype and the risk of breast cancer. Int J Cancer 2017;140:2657-66.

11 Kip KE, Marroquin OC, Kelley DE, et al. Clinical importance of obesity versus the metabolic syndrome in cardiovascular risk in women: a report from the women's ischemia syndrome evaluation (wise) study. Circulation 2004;109:706-13.

12 Wildman RP, Muntner P, Reynolds K, et al. The obese without cardiometabolic risk factor clustering and the normal weight with cardiometabolic risk factor clustering: prevalence and correlates of 2 phenotypes among the US population (NHANES 1999-2004). Arch Intern Med 2008;168:1617-24.

13 Karelis AD, St-Pierre DH, Conus F, et al. Metabolic and body composition factors in subgroups of obesity: what do we know? $J$ Clin Endocrinol Metab 2004;89:2569-75.

14 Karelis AD. Metabolically healthy but obese individuals. The Lancet 2008;372:1281-3.

15 Rey-López JP, de Rezende LF, Pastor-Valero M, et al. The prevalence of metabolically healthy obesity: a systematic review and critical evaluation of the definitions used. Obes Rev 2014;15:781-90.

16 Lin $\mathrm{H}$, Zhang $\mathrm{L}$, Zheng $\mathrm{R}$, et al. The prevalence, metabolic risk and effects of lifestyle intervention for metabolically healthy obesity: a systematic review and meta-analysis: a PRISMA-compliant article. Medicine 2017;96:e8838.

17 Ogorodnikova AD, Kim M, McGinn AP, et al. Incident cardiovascular disease events in metabolically benign obese individuals. Obesity 2012;20:651-9.

18 Ortega FB, Lee D-chul, Katzmarzyk PT, et al. The intriguing metabolically healthy but obese phenotype: cardiovascular prognosis and role of fitness. Eur Heart J 2013;34:389-97.

19 Yang HK, Han K, Kwon H-S, et al. Obesity, metabolic health, and mortality in adults: a nationwide population-based study in Korea. Sci Rep 2016;6. 
20 Primeau V, Coderre L, Karelis AD, et al. Characterizing the profile of obese patients who are metabolically healthy. Int $J$ Obes 2011;35:971-81.

21 Hinnouho G-M, Czernichow S, Dugravot A, et al. Metabolically healthy obesity and risk of mortality: does the definition of metabolic health matter? Diabetes Care 2013;36:2294-300.

22 Thomsen M, Nordestgaard BG. Myocardial infarction and ischemic heart disease in overweight and obesity with and without metabolic syndrome. JAMA Intern Med 2014;174:15-22.

23 Song Y, Manson JE, Meigs JB, et al. Comparison of usefulness of body mass index versus metabolic risk factors in predicting 10 -year risk of cardiovascular events in women. Am J Cardiol 2007;100:1654-8.

24 Calori G, Lattuada G, Piemonti L, et al. Prevalence, metabolic features, and prognosis of metabolically healthy obese Italian individuals: the Cremona study. Diabetes Care 2011;34:210-5.

25 Hinnouho G-M, Czernichow S, Dugravot A, et al. Metabolically healthy obesity and the risk of cardiovascular disease and type 2 diabetes: the Whitehall II cohort study. Eur Heart J 2015;36:551-9.

26 Kramer CK, Zinman B, Retnakaran R. Are metabolically healthy overweight andobesity benign conditions?: a systematic review and meta-analysis. Ann Intern Med 2013;159:758-69.

27 Fan J, Song Y, Chen Y, et al. Combined effect of obesity and cardio-metabolic abnormality on the risk of cardiovascular disease: a meta-analysis of prospective cohort studies. Int $\mathrm{J}$ Cardiol 2013;168:4761-8.

28 Eckel N, Meidtner K, Kalle-Uhlmann T, et al. Metabolically healthy obesity and cardiovascular events: a systematic review and metaanalysis. Eur J Prev Cardiol 2016;23:956-66.

29 Zheng R, Zhou D, Zhu Y. The long-term prognosis of cardiovascular disease and all-cause mortality for metabolically healthy obesity: a systematic review and meta-analysis. J Epidemiol Community Health 2016;70:1024-31.

30 Stefan N, Häring H-U, Schulze MB. Metabolically healthy obesity: the low-hanging fruit in obesity treatment? Lancet Diabetes Endocrinol 2018;6:249-58.

31 Moher D, Shamseer L, Clarke M, et al. Preferred reporting items for systematic review and meta-analysis protocols (PRISMA-P) 2015 statement. Syst Rev 2015;4:1.

32 Stefan N, Schick F, Häring H-U. Causes, characteristics, and consequences of metabolically unhealthy normal weight in humans. Cell Metab 2017;26:292-300.

33 Wells G, Shea B, O'Connell D, et al. The Newcastle-Ottawa scale (NOS) for assessing the quality of non randomised studies in meta-analyses. Available: http://www.ohri.ca/programs/clinical_ epidemiology/oxford.asp [Accessed 28 Jun 2019].

34 R Core Team. R: a language and environment for statistical computing $\mathrm{R}$ foundation for statistical computing, Vienna, Austria, Cited 2019. Available: www.Rproject.org

35 Viechtbauer W. Conducting Meta-Analyses in $R$ with the metafor Package. J Stat Softw 2010;36:1-48.

36 Higgins JPTet al. Measuring inconsistency in meta-analyses. BMJ 2003;327:557-60.

37 Mantel N, Haenszel W. Statistical aspects of the analysis of data from retrospective studies of disease. J Natl Cancer Inst 1959;22:719-48.

38 DerSimonian R, Laird N. Meta-Analysis in clinical trials. Control Clin Trials 1986;7:177-88.

39 Lassale C, Tzoulaki I, Moons KGM, et al. Separate and combined associations of obesity and metabolic health with coronary heart disease: a pan-European case-cohort analysis. Eur Heart $J$ 2018;39:397-406.

40 Lavie CJ, Laddu D, Arena R, et al. Healthy weight and obesity prevention: JACC health promotion series. J Am Coll Cardiol 2018;72:1506-31.

41 Ortega FB, Cadenas-Sanchez C, Migueles JH, et al. Role of physical activity and fitness in the characterization and prognosis of the metabolically healthy obesity phenotype: a systematic review and meta-analysis. Prog Cardiovasc Dis 2018;61:190-205.

42 Arnlöv J, Ingelsson E, Sundström J, et al. Impact of body mass index and the metabolic syndrome on the risk of cardiovascular disease and death in middle-aged men. Circulation 2010;121:230-6.

43 Lee SK, Kim SH, Cho G-Y, et al. Obesity phenotype and incident hypertension: a prospective community-based cohort study. $J$ Hypertens 2013;31:145-51.

44 Eckel N, Li Y, Kuxhaus O, et al. Transition from metabolic healthy to unhealthy phenotypes and association with cardiovascular disease risk across BMI categories in 90257 women (the Nurses' Health Study): 30 year follow-up from a prospective cohort study. Lancet Diabetes Endocrinol 2018;6:714-24.

45 Viechtbauer W, Cheung MW-L. Outlier and influence diagnostics for meta-analysis. Res Synth Method 2010;1:112-25.

46 Begg CB, Mazumdar M. Operating characteristics of a RANK correlation test for publication bias. Biometrics 1994;50:1088-101.

47 Egger M, Smith GD, Schneider M, et al. Bias in meta-analysis detected by a simple, graphical test. BMJ 1997;315:629-34.

48 Sterne JAC, Egger M, Smith GD. Systematic reviews in health care: investigating and dealing with publication and other biases in metaanalysis. BMJ 2001;323:101-5. 